\title{
Integrating Audio and Visual Cues for Speaker Friendliness in Multimodal Speech Synthesis
}

\author{
David House \\ Department of Speech, Music and Hearing \\ School of Computer Science and Communication, KTH, Stockholm, Sweden \\ davidh@speech.kth.se
}

\begin{abstract}
This paper investigates interactions between audio and visual cues to friendliness in questions in two perception experiments. In the first experiment, manually edited parametric audio-visual synthesis was used to create the stimuli. Results were consistent with earlier findings in that a late, high final focal accent peak was perceived as friendlier than an earlier, lower focal accent peak. Friendliness was also effectively signaled by visual facial parameters such as a smile, head nod and eyebrow raising synchronized with the final accent. Consistent additive effects were found between the audio and visual cues for the subjects as a group and individually showing that subjects integrate the two modalities. The second experiment used data-driven visual synthesis where the database was recorded by an actor instructed to portray anger and happiness. Friendliness was correlated to the happy database, but the effect was not as strong as for the parametric synthesis.
\end{abstract}

Index Terms: audio-visual speech perception, multimodal integration, human-machine interaction, audio-visual speech synthesis

\section{Introduction}

Asking questions to obtain information or to establish social interaction is one of the most fundamental functions of human dialogues. In human-machine spoken language interaction, asking questions is obviously important from both a user and a system perspective. For the user, asking for information is a natural and easy way of accessing data, while the ability of the system to handle questions is fundamental if the user is to be given the initiative. For the system, posing appropriate questions is necessary for guiding the user, for maintaining the dialogue and for error handling. However, question management is crucial not only for accessing information, but also for creating a smooth flow of the dialogue between the user and the system. In this regard, not only are informationrelated questions important, but also the management of social-related questions. There is now considerable evidence which suggests that we relate socially to computers and especially embodied computer agents in much the same way as we relate to other humans (e.g. [1][2][3]).

The variability of intonation in asking questions is also a topic of much interest. Not only does question intonation vary in different languages but also different types of questions (e.g. wh, yes/no or echo questions) can result in different kinds of question intonation [4]. In very general terms, the most commonly described tonal characteristics for questions are high final pitch and overall higher pitch [5]. In many languages, yes/no questions are reported to have a final rise, while wh-questions typically are associated with a final low.
Wh-questions can, however, often be associated with a large number of various contours [6]. In Swedish, an optional final rise can be of importance for signaling interrogative mode [7].

The prosodic features of the ends of phrases can also reveal characteristics about questions which are important for the dialogue flow. For example, there has been recent interest in the automatic analysis of phrase final tones and short utterances with the objective of categorizing and extracting dialogue acts such as agreement, acknowledgement, backchannels, turntaking and speaker attitude (see e.g. [8][9][10]).

In a recent study of phrase-final features in a set of 200 wh-questions extracted from a large corpus of computerdirected spontaneous speech in Swedish, it was found that final rises occurred in 22 percent of the utterances [11]. Moreover, there was an indication that the questions ending in a final rise were more oriented to signaling a social interest while those with a final low were more oriented to a request for specific information. These results are consistent with a study on German spontaneous speech in which Kohler [12] proposes that "rising pitch expresses friendliness, interest and openness towards the addressee, while falling pitch focuses on routine, lack of interest and categoricalness" (p. 207).

In an effort to explore the effects of audio-visual cues on the perception of question intonation in Swedish an experiment was carried out to test if visual cues such as a smile, vertical nods, eye narrowing and eyebrow lowering could influence the perception of question and statement intonation in Swedish [7]. Results showed only a marginal influence of the visual cues. While the hypothesized cues for declarative mode (smile, short head nod and eye narrowing) reinforced declarative intonation, the hypothesized cues for interrogative mode (slow head nod and eyebrow lowering) led to more ambiguity in the responses. Similar results were obtained for English by Srinivasan and Massaro [13]. Although they were able to demonstrate that the visual cues of eyebrow raising and head tilting synthesized based on a natural model reliably conveyed question intonation, their experiments showed a weak visual effect relative to a strong auditory effect of intonation.

In view of these results, it seemed worthwhile to explore the possibility of visual cues influencing the type or category of the question such as an information question or a social question, rather than using visual cues to change declarative intonation to an interrogative percept or vice-versa. Specifically, this paper builds on the results of [11] and investigates the effect of adding visual cues to the auditory stimuli which were judged as most friendly and least friendly in the previous study. The visual cues were manifested by a talking head using two types of audio-visual synthesis, namely parametric and data-driven visual synthesis. 


\section{Experiment 1 (parametric synthesis)}

\subsection{Method}

In the previous study [11], F0 peak location and F0 peak height were manipulated in the question "Vad heter du?" (What is your name?), which was also a common question posed by users when interacting with the animated agent in the dialogue system. In these experiments, a late, high peak was generally perceived as expressing more friendliness than an earlier, lower peak. Two representative stimuli from the earlier study were selected for the present study. The stimulus illustrated in Figure 1 was judged as least friendly while the one illustrated in Figure 2 was judged as most friendly.

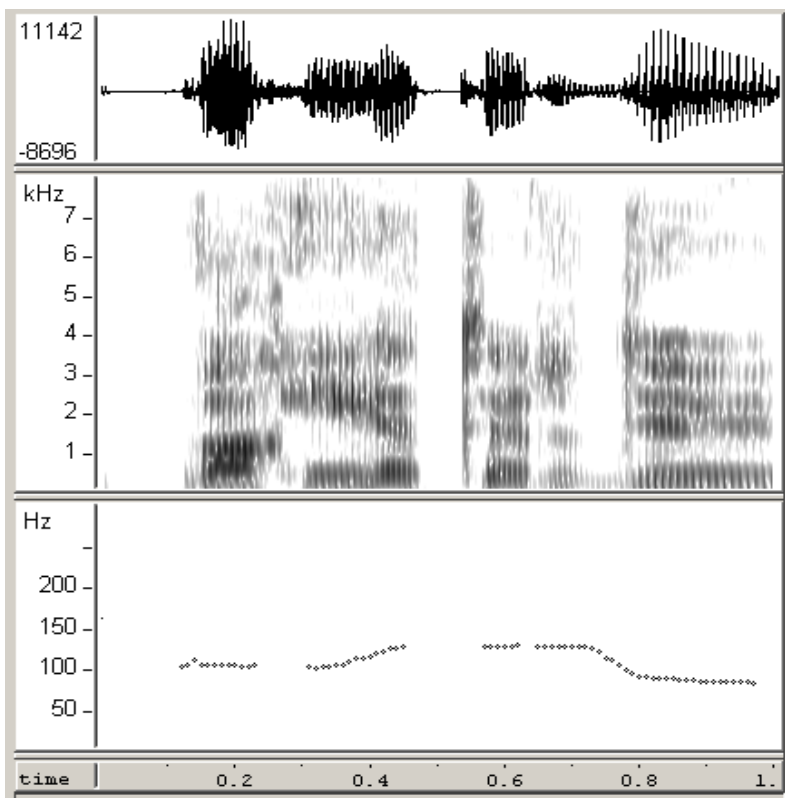

Figure 1: Waveform, spectrogram and F0 contour of the synthesized utterance "Vad heter du?" (What is your name?) with an early, low-peak focal accent on "heter".

In order to test the influence of visual cues on the perception of friendliness, two configurations combining different facial gestures were synthesized using an experimental version of the Infovox 330 diphone Swedish male MBROLA voice implemented as a plug-in to the WaveSurfer speech tool. The two configurations were designed to reinforce the two audio examples described above, the low peak in the early focal position (information-oriented) and the high peak in the late focal position (friendly/social-oriented). For the informationoriented configuration, an early nod and a lowering gesture of the eyebrows were synchronized with the early focal accent (F0 peak) on the second syllable. For the friendly/socialoriented configuration, a late nod and a raising gesture of the eyebrows were synchronized with the late focal accent (F0 peak) on the final syllable. In addition, a smile was added throughout the utterance with increasing amplitude at the end of the utterance after the nod. Samples of the configurations are shown in Figure 3.

The two audio configurations were combined with the two video configurations making four stimuli. The stimuli were then converted to video files. A perception test was carried out in which the files were played using Windows Media Player and projected onto a screen in a classroom.

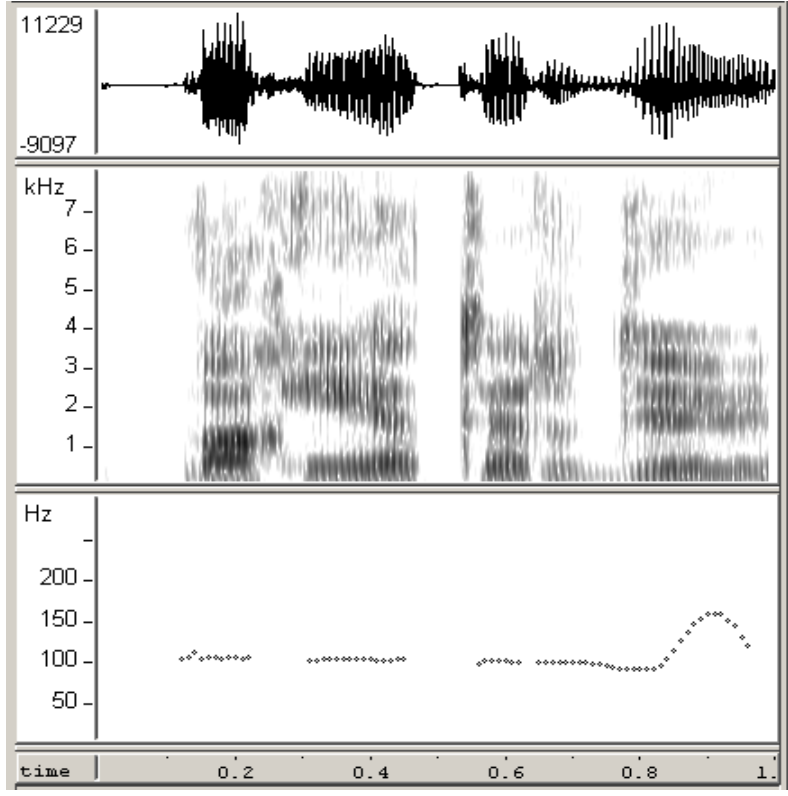

Figure 2: Waveform, spectrogram and F0 contour of the synthesized utterance "Vad heter du?" (What is your name?) with a late, high-peak focal accent on " $d u$ ".

The audio was played through high-quality loudspeakers. 27 native Swedish subjects were presented with three tokens of each of the four stimuli in random order. The subjects were asked to rate each stimulus on an unnumbered four-point scale where the endpoints were "friendly" and "less friendly." Each stimulus was played twice in succession.

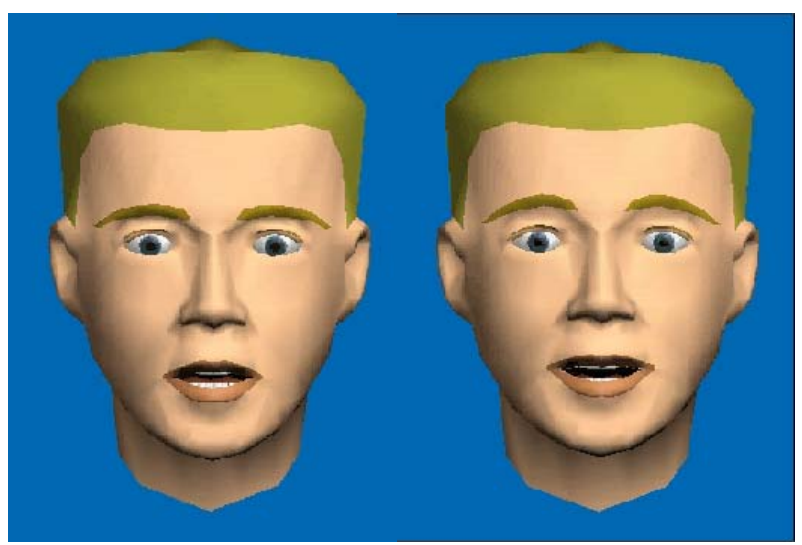

Figure 3: The hypothesized information-oriented configuration (left) and social/friendly configuration (right) sampled in the middle of the second vowel of the utterance "Vad heter du?" (What is your name?).

\subsection{Results}

The results showing mean scores for the subjects as a group are presented in Figure 4. It is clear that the consistent stimuli, where both audio and visual cues are intended to convey the same attitude, are perceived as conveying a low vs. a high degree of friendliness. For the inconsistent stimuli, the visual cues outweigh the auditory cues (i.e. the friendly face combined with the early, low peak received a higher rating of 
friendliness than did the info-face combined with the late, high peak).

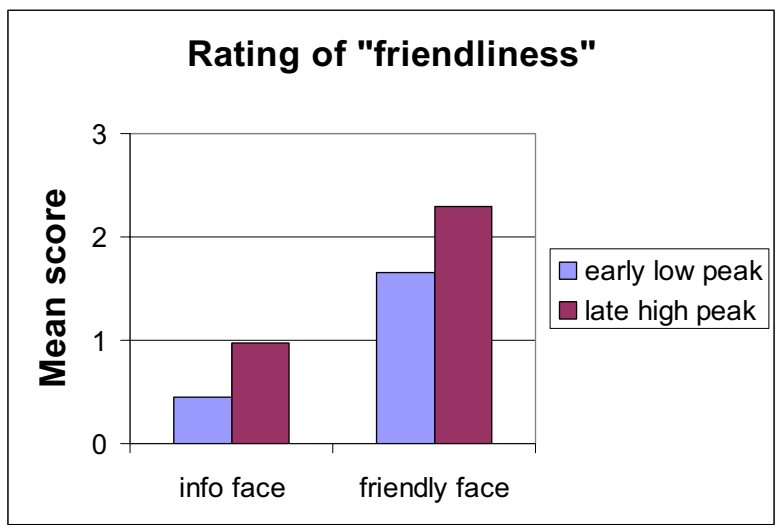

Figure 4: Results from the parametric synthesis test showing the mean "friendliness score" for each stimulus.

While these results tell us how the subjects performed as a group, they do not reveal if the individual subjects actually integrated the cues or if some of the subjects were more sensitive to visual cues and some were more sensitive to auditory cues. In order to examine each individual subject's responses, the individual responses were converted to rank order. In the event of a tie, the stimuli were each given the same score halfway between the two rankings. For 18 of the 27 subjects the stimuli were ranked in the following order from least friendly to most friendly: early low peak and infoface, late high peak and info-face, early low peak and friendly face, and late high peak and friendly face. This ranking comprised the median ranking for the group as shown in Figure 5.

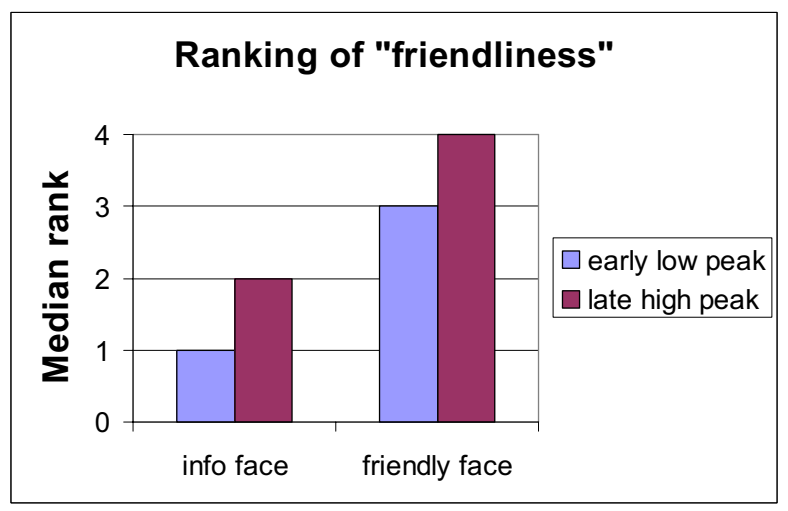

Figure 5: Results from the parametric synthesis test showing the median ranking of "friendliness" for each stimulus.

Two subjects showed no differences in ranking between different auditory stimuli for the same face configurations, and three subjects showed no difference in ranking between the different auditory stimuli for the info-face, but did rank the auditory stimuli differently for the friendly face. These results show evidence that most of the subjects integrated the auditory and visual modalities in perceiving friendliness. There were, however, a few subjects who showed more sensitivity to visual cues than to auditory cues.

\section{Experiment 2 (data-driven synthesis)}

\subsection{Method}

A different way of obtaining visual stimuli is by using datadriven visual synthesis. Facial movement data was collected by recording the positions of infrared markers on the face of an actor who was instructed to produce short sentences with different emotions [14]. The 3D coordinates for each marker were registered and this information was then used to drive a talking head based on the MPEG 4 facial animation standard [15]. Using the databases of different emotions results in talking head animations which differ in articulation and visual expression. For the current experiment, databases of angry, happy and neutral emotions were used to synthesize the same utterance as in the previous experiment, "Vad heter du?" (What is your name?). Samples of the three versions of the visual stimuli are presented in Figure 6. As in the previous experiment, the three versions of the visual synthesis were combined with two audio configurations: low, early pitch peak and high, late pitch peak resulting in six stimuli. There were some differences in the audio stimuli between experiment 1 and 2 in that for experiment 2 the stimuli were created using intonation rules resulting in a deaccentuation of "heter" in the temporal domain in the non-focal version. The intonation contours were otherwise very similar for the two experiments. A perception test using these six stimuli was carried out in the same way and on the same occasion as the previous experiment using the same 27 subjects.

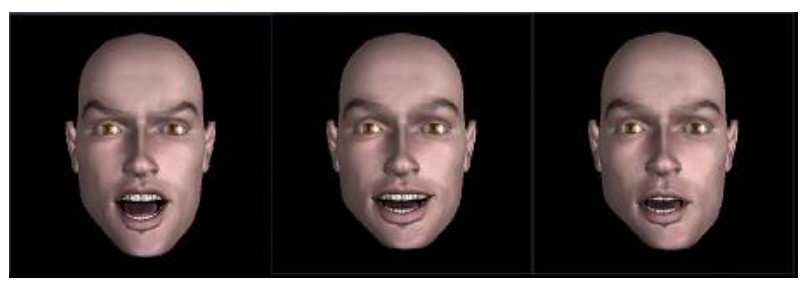

Figure 6. Visual stimuli generated by data-driven synthesis from the angry database (left), the happy database (middle) and the neutral database (right). All samples are taken from the middle of the second vowel of the utterance "Vad heter du?" (What is your name?).

\subsection{Results}

The results showing mean scores for the subjects as a group are presented in Figure 7. It is quite clear that the face synthesized from the angry database elicited the lowest friendliness score. However, there is still evidence of interaction from the audio, as the angry face with the late, high peak received a higher friendliness score than did the angry face with the early, low peak. The faces from the other databases (happy and neutral) elicited more friendliness responses, but neither combination of face and audio received as high a friendliness score as did the optimal stimulus from the parametric synthesis experiment.

As in experiment 1, the individual responses were converted to rank order. A greater variation in the order was apparent as shown in Figure 8. However, the happy face combined with the late high peak received the highest median rank of all the stimuli. 


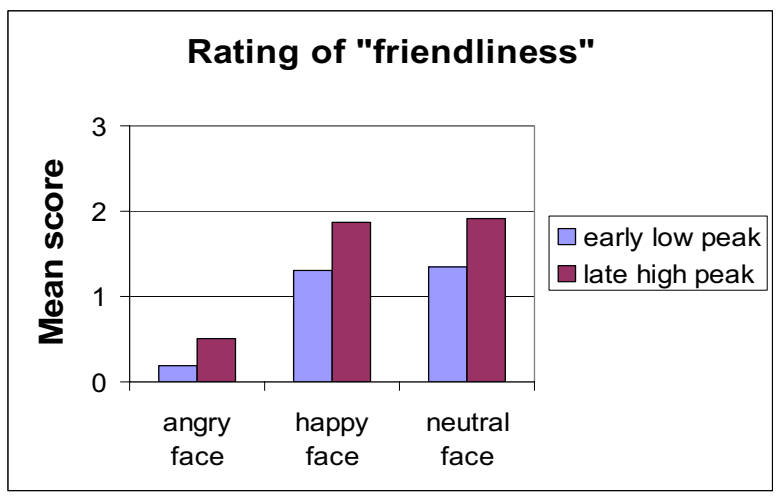

Figure 7: Results from the data-driven synthesis test showing the mean "friendliness score" for each stimulus.

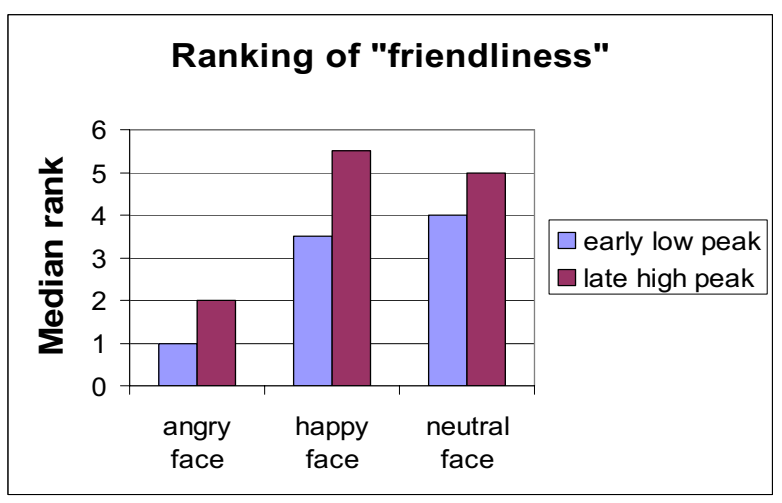

Figure 8: Results from the data-driven synthesis test showing the median ranking of "friendliness" for each stimulus.

\section{Discussion}

The results of the experiments presented in this paper present evidence of the interaction of audio and visual cues to the signaling of friendliness. While facial gestures in the visual modality were shown to be strong signals of friendliness, the effects of the auditory, focal accent cues for friendliness were clearly and consistently perceived in all the different versions of visual synthesis. This indicates that subjects make use of both modalities to make a judgement of speaker attitude and stresses the need to consider both the visual and audio aspects in expressive synthesis.

The results were clearer for the parametric synthesis than for the data-driven synthesis. This could partly be due to the fact that the parametric synthesis is more stereotypical, and when it successfully captures an attitude, it is perceived in clearer categorical terms. There may also be intrinsic differences in the way the two faces are perceived. In the data-driven synthesis, facial movement was limited mostly to the articulators (lips and mouth) and some eyebrow movement. The final smile and head movement present in the parametric synthesis was absent from the data-driven synthesis and may have contributed to the more successful "friendly" parametric face. It could be interesting and useful in future work to experiment with a combination of these techniques where for example head and eyebrow movement and the smile could be controlled within a data-driven visual synthesis framework.

\section{Acknowledgements}

This research was carried out with partial support from the MUSCLE Network of Excellence (NoE) funded by the European Commission under FP6 (Contract 507752). Special thanks to Jonas Beskow, Mikael Nordenberg and Magnus Nordstrand for creating the data-driven visual synthesis.

\section{References}

[1] Bell, L. and Gustafson, J. "Interaction with an animated agent in a spoken dialogue system", Proc. Eurospeech '99, Budapest, 1143-1146, 1999.

[2] Nass, C. and Moon, Y. "Machines and mindlessness: Social responses to computers", Journal of Social Issues 56 (1), 81-103, 2000.

[3] Brave, S., Nass, C., and Hutchinson, K. "Computers that care: investigating the effects of orientation of emotion exhibited by an embodied computer agent", International Journal of Human-Computer Studies 62, 161-178, 2005

[4] Ladd, D.R. Intonation phonology. Cambridge University Press, Cambridge. 1996.

[5] Hirst, D., and Di Cristo, A. "A survey of intonation systems", In D. Hirst and A. Di Cristo (eds.) Intonation Systems. Cambridge University Press, Cambridge, 1-45, 1998.

[6] Cruttenden, A. Intonation. Cambridge University Press, Cambridge, 1986.

[7] House, D. "Intonational and visual cues in the perception of interrogative mode in Swedish", Proc. of ICSLP 2002, Denver, Colorado, 1957-1960, 2002.

[8] Caspers, J. "On the function of low and high boundary tones in Dutch dialogue", Proc.15th ICPhS, Barcelona, 1771-1774, 2003.

[9] Cerrato, L. "Some characteristics of feedback expressions in Swedish", Proc. of Fonetik 2002, TMHQPSR 44, vol. 1, 101-104, 2002.

[10] Ferrer, L., Shriberg, E., and Stolcke, A., "Is the speaker done yet? Faster and more accurate end-of utterance detection using prosody", Proc. of ICSLP 2002, Denver, Colorado, 2061-2064, 2002.

[11] House, D. "Phrase-final rises as a prosodic feature in wh-questions in Swedish human-machine dialogue", Speech Communication 46, 268-283, 2005.

[12] Kohler, K.J. "Pragmatic and attitudinal meanings of pitch patterns in German syntactically marked questions", In G. Fant, H. Fujisaki, J. Cao and Y. Xu (Eds.) From traditional phonology to modern speech processing, Foreign Language Teaching and Research Press, Beijing, 205-214, 2004.

[13] Srinivasan, R.J., and Massaro, D.W., "Perceiving prosody from the face and voice: Distinguishing statements from echoic questions in English", Language and Speech 46(1), 1-22, 2003.

[14] Beskow J., Cerrato L., Granström B., House D., Nordstrand M., and Svanfeldt G. The Swedish PF-Star Multimodal Corpora. Proc LREC Workshop, Multimodal Corpora: Models of Human Behaviour for the Specification and Evaluation of Multimodal Input and Output Interfaces, Lisbon, 34-37, 2004.

[15] Beskow, J. and Nordenberg, M. "Data-driven synthesis of expressive visual speech using an MPEG-4 talking head”, Proc. Interspeech 2005, Lisbon, 793-796, 2005. 